\title{
The Newton-Wigner localization concept and noncommutative space
}

\author{
R. M. MIR-KaSimov \\ JINR, Bogoliubov Laboratory of Theoretical Physics, 141980 Dubna, Russia, \\ and \\ Department of Mathematics, Izmir Institute of Technology, 35430 Urla/Izmir, Turkey
}

In the formulation of the Newton-Wigner postulates for the relativistic localized states the hypothesis of commutativity of the position operator components is silently accepted as an evident fact. In the present work it is shown that commutativity is not necessary condition and the alternative (noncommutative) approach to the relativistic position operator and localization concept can be realized in a framework of the physically as well as mathematically comprehensive scheme.

PACS: 02.20.Uw, 02.20.-a

Key words: localization, noncommutative, relativistic

\section{Introduction}

The concept of localization is one of the most important and most intriguing problems in quantum theory. The idea of localizability plays principal role in the physical interpretation of the theory. We can not avoid this concept when comparing the measurement results with the theory predictions and considering the uncertainty relations. The localizability is necessary element in constructing the initial and final states when describing the collision phenomena. In the case of the two-body problem the question of the localizability of the bound state must be solved in a transparent way.

In the non-relativistic case for the potentials $V(r)$ depending only on relative distance between interacting particles $r=|\mathbf{r}|$ the coordinates of the center of mass $\mathbf{R}$ and $\mathbf{r}$ are separated. The Galilean invariance of the motion of the system as a whole is respected, the free motion of the bound state (of the system as the whole) is described by the irreducible unitary representation of the Galilean group. From this point of view it would be natural to call the spherically symmetric potentials $V(r)$ the Galilean potentials. The internal motion of the system is reduced to the motion of the effective particle with the reduced mass $\mu=\frac{m_{1} m_{2}}{m_{1}+m_{2}}$ in the field of potential. We can call the two-body systems with the spherically symmetric potentials $V(r)$ the Galilean elementary systems by evident analogy with the relativistic particle localization concept of E. Wigner [2]. Let us remember also that non-relativistic compound quark models of hadrons (with spherically symmetric potentials) are very efficient, and they describe the bound states which are the elementary systems. But there are no doubts that these compound systems are actually relativistic and it is necessary to find the comprehensive relativistic potential theory standing behind. 
We stress that keeping in mind the potential relativistic potential models we seek for such relativistic analog of the relative coordinate $\mathbf{r}$ on which the interaction potential depends so that the total relativistic invariance is respected in analogy with Galilean invariance of the non-relativistic two-particle problem with the spherically symmetric potentials $V(r)$.

This discussion can be continued but it is clear that old problem of finding the relativistic position operator still deserves to search for its solution. The basic ideas on this subject have bee expressed by Newton and Wigner $[2]^{1}$ ). Their essential result is that for single particles a notion of the localizability and a corresponding commuting observables are uniquely determined by relativistic kinematics. On the other hand no relativistic quantum theory of interaction based on these ideas was constructed. In the present contribution we shall consider the possibility to introduce the concept of the non-commuting relativistic position operators obeying all Newton-Wigner postulates, having the transparent physical interpretation and admitting very simple quantum dynamical interpretation.

It must be stressed that the standard quantum-mechanical position operator $\hat{\mathbf{x}}=\mathrm{i} \hbar \nabla_{\mathbf{p}}$ is connected with the Euclidean structures in terms of which the localization of a particle is considered. Let us quote here [2]: "Existence and uniqueness of a notion of localizability for a physical system are properties which depend only on the transformation law of the system under Euclidean group, i.e., the group of all space translations and rotations. The analysis of localizability in the Lorentz and Galilei invariant cases is then just a matter of discussing what representations of the Euclidean group can arise there". Both groups-Galilean and Poincarécontain the Euclidean group as their subgroup. But might be there are another realizations of the Euclidean group in the framework of the representation theory which allows another definition of the position operator? We try to show here that the answer is positive.

The fact that the manifold of the physically realizable states contains only solutions with the positive energy has a number of consequences for the observables. Consider the solutions of the Klein-Gordon equation $\varphi, \psi$ :

$$
\varphi, \psi \in\left\{(+): p^{\mu} p_{\mu}=\left(p^{0}\right)^{2}-\tilde{p}^{2}=m^{2} c^{2}, \quad p^{0} \geq 0\right\}
$$

with the inner product

$$
(\varphi, \psi)=\int_{(+)} \mathrm{d} \Omega_{\mathbf{p}} \overline{\varphi(\mathbf{p})} \psi(\mathbf{p}), \quad \mathrm{d} \Omega_{\mathbf{p}}=\frac{\mathrm{d} \mathbf{p} m c}{p^{0}} .
$$

The standard position operator

$$
\hat{\hat{\mathbf{x}}}=\mathrm{i} \hbar \nabla_{p}
$$

is non-hermitian in the metric (2):

1) There exists a huge literature on the subject, see e.g. $[4,12]$ and references therein. 


$$
\begin{aligned}
(\varphi, \hat{\hat{\mathbf{x}}} \psi) & =\int_{(+)} \mathrm{d} \Omega_{\mathbf{p}} \overline{\varphi(\mathbf{p})} \mathrm{i} \hbar \nabla_{p} \psi(\mathbf{p})= \\
& =\int_{(+)} \mathrm{d} \Omega_{\mathbf{p}} \overline{\left[\left(\mathrm{i} \hbar \nabla_{p}-\frac{\mathrm{i} \hbar \mathbf{p}}{\mathbf{p}^{2}+m^{2} c^{2}}\right) \varphi(\mathbf{p})\right]} \psi(\mathbf{p}) .
\end{aligned}
$$

So the operator $i \hbar \nabla_{p}$ does not correspond to any observable and can no be interpreted as an physical operator. It follows also that the Klein-Gordon wave function can not be considered as an probability amplitude to find the particle at the point $\mathbf{x}$ at the moment of time $x^{0}$.

The simplest way to obtain the position operator is to accept that the position operator is the hermitian part of $\hat{\mathbf{x}}=\mathrm{i} \hbar \nabla_{p}$ :

$$
\hat{\mathbf{x}}_{\mathrm{NW}}=\frac{1}{2}\left[\hat{\mathbf{x}}+\hat{\mathbf{x}}^{\dagger}\right]=\mathrm{i} \hbar \nabla_{p}-\frac{\mathrm{i} \hbar}{2} \frac{\mathbf{p}}{\mathbf{p}^{2}+m^{2} c^{2}} .
$$

Newton and Wigner derived this operator on a basis of a number of conditions which localized states must satisfy (see [1]).

For the wave function of the localized state (at the moment $x^{0}$ ) $\psi_{\mathbf{y}}(\mathbf{x})$ in the configurational space we obtain.

A number of conclusions can be derived from the Newton-Wigner theory. We indicate two of them:

First: The position operator components $\hat{x}_{N W}^{i}$ commute

$$
\left[\hat{x}_{\mathrm{NW}}^{i}, \hat{x}_{\mathrm{NW}}^{j},\right]=0, \quad\left[\hat{x}_{\mathrm{NW}}^{i}, p^{j},\right]=\mathrm{i} \delta_{i j} .
$$

Second: The localized eigenfunction is not $\delta(\mathbf{x}-\mathbf{y})$ as in the non-relativistic theory, it is a function $\psi_{\mathbf{y}}(\mathbf{x})$ smeared in the spatial region of the size of the Compton wave length of the particle $\lambda_{0}$, because $\delta(x-y)$ can't be constructed from the positive frequency solutions only:

$$
\psi_{\mathbf{y}}(\mathbf{x})=\operatorname{const}\left(\frac{m c}{\hbar r}\right)^{5 / 4} K_{5 / 4}\left(\frac{r}{\lambda_{0}}\right), \quad r=|\mathbf{x}-\mathbf{y}|, \quad \lambda_{0}=\frac{\hbar}{m c},
$$

$K_{\nu}(z)$ is MacDonald's function, $\lambda_{0}$ is Compton wave length of the particle.

The content of this article is as follows. In Sect. 2 we consider the noncommutative alternative to the Newton-Wigner coordinate and related concept of the relativistic configurational space. In Sect. 3 the two-body problem in the relativistic configurational space is formulated.

\section{Alternative to Newton-Wigner approach}

The Newton-Wigner theory uses essentially the momentum space. To determine the nonlocal operator $\hat{\mathbf{x}}_{\mathrm{NW}}(5)$ directly in the configurational space would be very difficult. 
But there is another circumstance essential for formulating the main idea of the present paper. In [1] the wave functions localized at different points are connected by translation:

$$
\mathbf{x} \longrightarrow \mathbf{x}+\mathbf{a}, \quad \mathrm{e}^{\mathrm{ik}(\mathbf{x}+\mathbf{a})}=\mathrm{e}^{\mathrm{ik \mathbf {kx }}} \mathrm{e}^{\mathrm{ika}} .
$$

The second relation has two mathematical meanings.

1. We consider, as in [1] the translations in the configurational space. Then the plane waves (exponentials) are the matrix elements of the irreducible unitary representations of the translation group numbered by the value of momentum $\mathbf{k}$. Fourier transformation is the expansion in matrix elements of the unitary irreducible representations of the translation group of the configurational space.

2. We consider (in contrast to [1]) the translations in the momentum $\mathbf{k}$-space. Then the same formula (8) describes the matrix element of the product of two irreps numbered by $\mathrm{x}$ and a correspondingly by the vector (of the momentum space) $\mathbf{k}$. The inverse Fourier transformation is the expansion in matrix elements of the unitary irreducible representations of the translation group of the momentum space space.

Such a symmetry between transformation within the same representation and the product of the representations is specific to the Euclidean translations. In the non-relativistic theory the difference between 1 . and 2 . is formal and unimportant because the geometries of the configurational and momentum spaces are isomorphic (mathematically) and Euclidean. Physical sense of the configurational and momentum spaces is different of course. The translations of the momentum space corresponds to Galilean transformations:

$$
\begin{aligned}
\mathbf{x} & \longrightarrow \mathbf{x}+\mathbf{V} t \\
\dot{\mathbf{x}} & \longrightarrow \dot{\mathbf{x}}+\mathbf{V}, \\
m \dot{\mathbf{x}} & \longrightarrow m \dot{\mathbf{x}}+m \mathbf{V} \\
\mathbf{p} & \longrightarrow \mathbf{p}+\mathbf{k}, \quad \mathbf{p}=m \dot{\mathbf{x}}, \quad \mathbf{k}=m \mathbf{V} .
\end{aligned}
$$

The position operator (4) is the generator of translations of the momentum space.

Now we formulate the alternative to the Newton-Wigner concept. It is based on the simple observations.

1. From (4) we conclude that the geometry of the momentum space i.e. the manifold of realizable states of the relativistic particle of the positive frequency is the Lobachevsky space (1). We shall develop the one particle relativistic theory accepting this as the triggering point. Then we must substitute;

2. Galilean group $\longrightarrow$ Lorentz group;

3. Galilean boosts $\longrightarrow$ Lorentz boosts

$$
\hat{\mathbf{x}}=\mathrm{i} \hbar \nabla_{p} \longrightarrow \hat{\mathbf{x}}_{\mathrm{rel}}
$$


where

$$
\hat{\mathbf{x}}_{\mathrm{rel}}=\mathrm{i} \hbar \sqrt{1+\frac{\mathbf{p}^{2}}{m^{2} c^{2}}} \nabla_{p} .
$$

Thereby we consider (11) as the candidates for the relativistic position operators. But these are non-commuting operators. So proceeding along this geometrically natural way first what we must do is to give up with the commutativity of components of the position operator. We stress that in [1] the commutativity requirement is tacitly contained in the list of basic natural requirements.

First of all we note immediately that operators (11) are hermitian with the norm (2). After lifting the commutativity condition we can say that that the new position operators are (the simplest!) hermitian operators in this metric. Now in contrast with the commutative case the components of the position operator can not be measured. But no limitations exist that the very concept of the configurational space in the relativistic case can be modified as compared with the non-relativistic theory. The consequence of such a modification must be the change of the all concept of the measuring the position, uncertainty relations etc.

To make this statements more clear, let us return for a time being to the nonrelativistic case. As coordinates commute, we can diagonalize simultaneously all three components of it.

At the same time many other operators of the universal enveloping algebra of the Euclidean Lie algebra also are diagonal. For example the Casimir operator $\hat{\mathbf{x}}^{2}$ which is invariant operator of the Euclidean group of the momentum space

$$
\begin{aligned}
{\left[\hat{\mathbf{x}}^{2}\right] \mathrm{e}^{\mathrm{i} \mathbf{p x}} } & =\Delta_{\mathbf{p}} \mathrm{e}^{\mathrm{i} \mathbf{p x}}=\mathbf{x}^{2} \mathrm{e}^{\mathrm{i} \mathbf{p x}}, \\
\hat{x}^{i} \mathrm{e}^{\mathrm{i} \mathbf{p x}} & =x^{i} \mathrm{e}^{\mathrm{i} \mathbf{p x}}, \quad 0 \leq x<\infty, \quad-\infty<x^{i}<\infty
\end{aligned}
$$

Important is that the common eigenfunctions of these operators $e^{i p x}$ are the kernels of the Fourier transform connecting the Euclidean momentum space of the non-relativistic quantum mechanics and corresponding configurational space.

In the relativistic case it is natural to consider as the momentum space adequate from the physical point of view the space given by (1), i.e. the Lobachevsky space of the physical solutions of the Klein-Gordon equation. Integration over this space (with the Lorentz-invariant volume element $\mid D \Omega_{\mathbf{p}}$ ) is given by (2). If we wish to follow the concept presented in the previous paragraph we should consider the universal enveloping algebra of the Lorentz group., determine the maximal set of mutually commuting operators, determine their common eigenfunctions (new plane waves) and spectrum. The Casimir operator of the Lorentz group Lie algebra can be chosen in the form

$$
\hat{r}^{2}=\hat{\mathbf{x}}_{\mathrm{rel}}^{2}-\frac{\mathbf{M}^{2}}{m^{2} c^{2}}-\frac{\hbar^{2}}{m^{2} c^{2}},
$$

where $\mathbf{M}$ is the angular momentum operator. The non-relativistic limit of (13) is $\hat{\mathbf{x}}^{2}$ (see (12)). Spectrum of $r$ for the unitary representations takes continuous and discrete values. All these representations find the applications in various models of 
relativistic interactions. We shall concentrate on the so called principal series for which $0 \leq r<\infty$.

The eigenfunctions of $\hat{r}^{2}$ are the matrix elements of unitary irreducible representations of the Lorentz group or their generating functions--kernels of GelfandGraev transformations:

$$
\hat{r}^{2}\langle\mathbf{p} \mid \mathbf{r}\rangle=r^{2}\langle\mathbf{p} \mid \mathbf{r}\rangle, \quad\langle\mathbf{r} \mid \mathbf{p}\rangle=\langle\mathbf{p} \mid \mathbf{r}\rangle^{*} .
$$

They play the role of plane waves in the given relativistic formalism. Explicitly

$$
\langle\mathbf{r} \mid \mathbf{p}\rangle=\left(\frac{p^{0}-\mathbf{p n}}{m c}\right)^{-1-\mathrm{i} r m c / \hbar}, \quad \mathbf{n}^{2}=1
$$

The unit vector $\mathbf{n}$ gives the sense to the symbol $\mathbf{r}$-by definition

$$
\mathbf{r}=r \mathbf{n}
$$

We shall call the space of vectors $\mathbf{r}$ the relativistic configurational space ${ }^{2}$ ) The partial expansion for the plane wave (15) is

$$
\begin{aligned}
\langle\mathbf{r} \mid \mathbf{p}\rangle & =\sum_{l=0}^{\infty} \mathrm{i}^{l}(2 l+1) p_{l}(\cosh \chi, r) P_{l}\left(\mathbf{n}_{p} \cdot \mathbf{n}\right), \\
p^{0} & =\cosh \chi, \quad \mathbf{p}=\sinh \chi \mathbf{n}_{p}, \quad \mathbf{n}_{p}^{2}=1,
\end{aligned}
$$

where

$$
p_{l}(\cosh \chi, r)=(-1)^{l} \sqrt{\frac{\pi}{2 \sinh \chi}} \frac{\Gamma(\mathrm{i} r+l+1)}{\Gamma(\mathrm{i} r+1)} P_{-1 / 2+\mathrm{i} r}^{-1 / 2+\mathrm{i} r} \cosh \chi .
$$

The expansion (17) is analogous to the non-relativistic one

$$
\mathrm{e}^{\mathrm{i} \mathbf{p r}}=\sum_{l=0}^{\infty} \mathrm{i}^{l}(2 l+1) j_{l}(p r) P_{l}\left(\mathbf{n}_{p} \cdot \mathbf{n}\right)
$$

where $j_{l}(p r)=\sqrt{\frac{\pi}{2 p r}} J_{l+1 / 2}$ are the spherical Bessel functions. In the non-relativistic limit

$$
p_{l}(\cosh \chi, r) \longrightarrow j_{l}(p r) .
$$

The orthogonality and completeness conditions for the relativistic plane waves are

$$
\begin{aligned}
& \frac{1}{(2 \pi)^{3}} \int\langle\mathbf{r} \mid \mathbf{p}\rangle\left\langle\mathbf{p} \mid \mathbf{r}^{\prime}\right\rangle \mathrm{d} \Omega_{\mathbf{p}}=\delta\left(\mathbf{r}-\mathbf{r}^{\prime}\right), \\
& \frac{1}{(2 \pi)^{3}} \int\langle\mathbf{p} \mid \mathbf{r}\rangle\left\langle\mathbf{r} \mid \mathbf{p}^{\prime}\right\rangle \mathrm{d} \mathbf{r}=\delta\left(\mathbf{p}-\mathbf{p}^{\prime}\right)=\delta\left(\mathbf{p}-\mathbf{p}^{\prime}\right) \frac{p^{0}}{m \boldsymbol{c}} .
\end{aligned}
$$

\footnotetext{
${ }^{2}$ ) The concept of the relativistic configurational space have bbeen introduced in [6] (see also $[5]$ ), for further references see [7-9].
} 
The relativistic configurational space is an example of the quantum 3-dimensional Euclidean space. The quantum nature of the $\mathbf{r}$-space is predefined by the fact that the the Lie algebra of its isometry group is realized in a framework of noncommutative differential calculus. The momentum operators (generators of translations) are

$$
\begin{aligned}
& H_{0}=\hat{p}^{0}=\cosh \left(\mathrm{i} \frac{\partial}{\partial r}\right)+\frac{\mathrm{i}}{r} \sinh \left(\mathrm{i} \frac{\partial}{\partial r}\right)-\frac{\triangle_{\vartheta, \psi}}{2 r^{2}} \exp \left(\mathrm{i} \frac{\partial}{\partial r}\right), \\
& \hat{p}^{1}=-\sin \vartheta \cos \psi\left[\exp \left(\mathrm{i} \frac{\partial}{\partial r}\right)-H_{0}\right]-\mathrm{i}\left(\frac{\cos \vartheta \cos \psi}{r} \frac{\partial}{\partial \vartheta}-\frac{\sin \psi}{r \sin \vartheta} \frac{\partial}{\partial \psi}\right) \exp \left(\mathrm{i} \frac{\partial}{\partial r}\right), \\
& \hat{p}^{2}=-\sin \vartheta \sin \psi\left[\exp \left(\mathrm{i} \frac{\partial}{\partial r}\right)-H_{0}\right]-\mathrm{i}\left(\frac{\cos \vartheta \sin \psi}{r} \frac{\partial}{\partial \vartheta}+\frac{\cos \psi}{r \sin \vartheta} \frac{\partial}{\partial \psi}\right) \exp \left(\mathrm{i} \frac{\partial}{\partial r}\right), \\
& \hat{p}^{3}=-\cos \vartheta\left[\exp \left(\mathrm{i} \frac{\partial}{\partial r}\right)-H_{0}\right]+\mathrm{i} \frac{\sin \vartheta}{r} \frac{\partial}{\partial \vartheta} \exp \left(\mathrm{i} \frac{\partial}{\partial r}\right) .
\end{aligned}
$$

They play the role of inner derivatives in relevant differential calculi. These operators mutually commute

$$
\left[\hat{p}^{\mu}, \hat{p}^{\nu}\right]=0, \quad \mu, \nu=0,1,2,3 .
$$

But the corresponding differentials of the coordinate functions don't commute with the coordinate functions themselves. For the details we refer the reader to [9-11]. Note that that the integration in the second formula in (21) is carried over with the Euclidean volume element dr.

The common eigenfunctions of $\hat{p}^{\mu}$ are $\langle\mathbf{r} \mid \mathbf{p}\rangle(14)$

$$
\hat{p}^{\mu}\langle\mathbf{r} \mid \mathbf{p}\rangle=p^{\mu}\langle\mathbf{r} \mid \mathbf{p}\rangle,
$$

from which we conclude that the "plane waves" (14) indeed describe the free relativistic motion with definite value of the 4 -momentum. This is a new realization of the Lie algebra of the Euclidean group which we discussed in the Introduction.

Operators $\hat{p}^{\mu}$ identically satisfy the relativistic relation between energy and momentum (1). Important is also to note that these operators solve the problem of "extracting the root square" in the relation $\hat{p}^{\mu}=\sqrt{\mathbf{p}^{2}+m^{2} c^{2}}$ :

$$
\hat{p}^{0}\langle\mathbf{r} \mid \mathbf{p}\rangle=p^{0}\langle\mathbf{r} \mid \mathbf{p}\rangle=\sqrt{\mathbf{p}^{2}+m^{2} c^{2}}\langle\mathbf{r} \mid \mathbf{p}\rangle \text {. }
$$

In the non-relativistic limit

$$
|\mathbf{p}| \ll m c, \quad p^{0} \cong m c+\frac{\mathbf{p}^{2}}{2 m c}, \quad r \gg \frac{\hbar}{m c}
$$

relativistic plane waves $\langle\mathbf{r} \mid \mathbf{p}\rangle$ transfer to usual plane waves

$$
\begin{aligned}
\langle\mathbf{r} \mid \mathbf{p}\rangle & =\exp \left[-\left(1+\mathrm{i} r \frac{m c}{\hbar}\right) \ln \left(\frac{p^{0}-\mathbf{p n}}{m c}\right)\right] \cong \\
& \cong \exp \left[-\left(1+\mathrm{i} r \frac{m c}{\hbar}\right) \ln \left(1-\frac{\mathbf{p n}}{m c}+\frac{\mathbf{p}^{2}}{2 m^{2} c^{2}}+\ldots\right)\right] \cong \\
& \cong \exp \left(\mathrm{i} \frac{\mathbf{p} \cdot(r \mathbf{n})}{\hbar}\right)=\exp \left(\mathrm{i} \frac{\mathbf{p r}}{\hbar}\right) .
\end{aligned}
$$


The wave function of the particle can be expanded in the Fourier integral in the relativistic plane waves

$$
\psi(\mathbf{r})=\frac{1}{(2 \pi)^{3 / 2}} \int\langle\mathbf{r} \mid \mathbf{p}\rangle \psi(\mathbf{p}) \mathrm{d} \Omega_{\mathbf{p}} .
$$

Particles are localized in the relativistic configurational space in a usual sense. The position operator $\hat{\mathbf{r}}$ in $\mathbf{r}$-representation acts on a wave function in a usual way

$$
\hat{\mathbf{r}} \psi(\mathbf{r})=\mathbf{r} \psi(\mathbf{r})
$$

The eigenfunctions $\psi_{\mathbf{r}_{0}}(\mathbf{r})$ of $\hat{\mathbf{r}}$ corresponding to the eigenvalue $\mathbf{r}_{0}$ are $\psi_{\mathbf{r}_{0}}(\mathbf{r})=$ $\delta\left(\mathbf{r}-\mathbf{r}_{0}\right)$ so that

$$
\hat{\mathbf{r}} \psi_{\mathbf{r}_{0}}(\mathbf{r})=\mathbf{r} \psi_{\mathbf{r}_{0}} .(\mathbf{r})
$$

Eigenfunctions corresponding to different eigenvalues-i.e. the states localized at different points $\mathbf{r}_{0}$ and $\widetilde{\mathbf{r}}_{0}$-are orthogonal

$$
\int \psi_{\mathbf{r}_{0}} \psi_{\mathbf{r}_{0}} \mathrm{~d} \mathbf{r}=\delta\left(\widetilde{\mathbf{r}}_{0}-\mathbf{r}_{0}\right)
$$

which is the usual localization condition in the new relativistic configurational space.

\section{Relativistic two-body problem.}

In the relativistic configurational $\mathbf{r}$-space no local addition theorem like (8) exists and we must use the expansion (17). From this expansion the following "nonlocal" addition theorem follows [6]

$$
\int\left\langle\mathbf{r} \mid \mathbf{p}_{1}\right\rangle\left\langle\mathbf{p}_{2} \mid \mathbf{r}\right\rangle \mathrm{d} \mathbf{n}=\int\left\langle\mathbf{r} \mid \mathbf{p}_{1}(-) \mathbf{p}_{2}\right\rangle \mathrm{d} \mathbf{n},
$$

where $\mathbf{q}=\mathbf{p}_{1}(-) \mathbf{p}_{2}$ is a vector $\mathbf{p}_{1}$ boosted into the Lorentz frame moving with the velocity $\mathbf{v}=\frac{\mathbf{p}_{2} c}{\sqrt{\mathbf{p}_{2}^{2}+m^{2} c^{2}}}$

$$
\begin{aligned}
\mathbf{q} & =\mathbf{p}_{1}(-) \mathbf{p}_{2}, \\
\left(\mathbf{p}_{1}(-) \mathbf{p}_{2}\right)_{0} & =\left(\cosh \chi_{1} \cosh \chi_{2}-\sinh \chi_{1} \sinh \chi_{2}\left(\mathbf{n}_{p_{1}} \cdot \mathbf{n}_{p_{2}}\right)\right) .
\end{aligned}
$$

Of course for the standard plane waves the integral addition theorem like (32) is valid

$$
\int \exp \left(\mathbf{i p}_{1} \frac{m_{2}}{M} \mathbf{r}\right) \cdot \exp \left(-\mathrm{i} \mathbf{p}_{2} \frac{m_{1}}{M} \mathbf{r}\right) \mathrm{d} \mathbf{n}=\int \mathrm{e}^{\mathrm{i} \mathbf{p r}} \mathrm{d} \mathbf{n} .
$$

The formula (34) becomes necessary if we wish to multiply not the exponents themselves but their partial expansions (19). We see that the integration over $d \mathbf{n}$ is necessary to restore the partial expansion

$$
\mathrm{e}^{\mathrm{i} \mathbf{p r}}=\sum_{l=0}^{\infty} \mathrm{i}^{l}(2 l+1) j_{l}\left(\left|\frac{m_{2} \mathbf{p}_{1}-m_{1} \mathbf{p}_{2}}{M}\right|\right) P_{l}\left(\mathbf{n}_{p} \cdot \mathbf{n}\right)
$$


in the right hand side of (34). But such angular averaging commutes with the Galilean Hamiltonians (spherically symmetric potentials)

$$
\int\left[\frac{\mathbf{p}^{2}}{2 \mu}+V(r)\right] \psi(\mathbf{r}) \mathrm{d} \mathbf{n}=\left[\frac{\mathbf{p}^{2}}{2 \mu}+V(r)\right] \int \psi(\mathbf{r}) \mathrm{d} \mathbf{n} .
$$

Transferring to the relativistic two-body problem we must first note that hyperboloids in the momentum space corresponding to different particles are different, see (1). This must be taken into account for example in (15):

$$
\left\langle\mathbf{r} \mid \mathbf{p}_{i}\right\rangle=\left(\cosh \chi_{i}-\sinh \chi_{i}\left(\mathbf{n}_{p_{i}} \cdot \mathbf{n}\right)\right)^{-1-i r m_{i} c / \hbar}, \quad i=1,2,
$$

where $r$ is the (dimensional) analog of the relative distance between particles in the relativistic configurational space.

Thus the free relativistic two body wave function $\phi_{\mathrm{eff}}^{(r, 0)}(\mathbf{r})$ can be chosen in a form

$$
\phi_{\mathrm{eff}}^{(r, 0)}(\mathbf{r})=\left\langle\frac{m_{2}}{M I} \mathbf{r} \mid \mathbf{p}_{1}\right\rangle\left\langle\mathbf{p}_{2} \mid \frac{m_{1}}{M} \mathbf{r}\right\rangle
$$

It describes the free motion in the $\mathrm{CM}$ system, the relativistic free motion (see Sec. 2), and has the right non-relativistic limit. There are several possibilities to generalize for the relativistic case the formula (34) but our choice is the simplest from the formal point of view and most transparent from the physical point of view. In explicit form

$$
\begin{aligned}
\phi_{\mathrm{eff}}^{(r, 0)}(\mathbf{r})= & \left(\cosh \chi_{1}-\sinh \chi_{1}\left(\mathbf{n}_{p_{1}} \cdot \mathbf{n}\right)\right)^{-1-\mathrm{i}\left(m_{2} / M\right)\left(r m_{1} c / \hbar\right)} \times \\
& \times\left(\cosh \chi_{2}-\sinh \chi_{2}\left(\mathbf{n}_{p_{2}} \cdot \mathbf{n}\right)\right)^{-1+\mathrm{i}\left(m_{1} / M\right)\left(r m_{2} c / \hbar\right)}
\end{aligned}
$$

Now we apply the addition theorem (32) and obtain

$$
\int\left\langle\frac{m_{2}}{M} \mathbf{r} \mid \mathbf{p}_{1}\right\rangle\left\langle\mathbf{p}_{2} \mid \frac{m_{1}}{M} \mathbf{r}\right\rangle \mathrm{d} \mathbf{n}=\int\langle\mathbf{r} \mid \mathbf{q}\rangle \mathrm{d} \mathbf{n},
$$

where $q$ is given by (33). Remarkable is that the mass entering the expression for the relativistic plane wave in the right hand side of (40) is the reduced mass $\mu$

$$
\langle\mathbf{r} \mid \mathbf{q}\rangle=\left(\cosh \chi_{q}-\sinh \chi_{q}\left(\mathbf{n}_{q} \cdot \mathbf{n}\right)\right)^{-1-i r \mu c / \hbar} .
$$

We shall consider (41) as the free relativistic effective wave function describing the relative motion. In the presence of potential the $\phi_{\text {eff }}^{(0)}(\mathbf{r})$ is modified and we have

$$
\phi_{\mathrm{eff}}^{(r)}(\mathbf{r})=\frac{1}{(2 \pi)^{3 / 2}} \int\langle\mathbf{r} \mid \mathbf{k}\rangle \phi_{\mathrm{eff}}^{(r)}(\mathbf{k}) \mathrm{d} \Omega \mathbf{k} .
$$

Now we consider the arbitrary frame of reference. In absence of the external field the our 2-body system moves with the constant velocity. This allows us to 
write the relativistic 2-body wave function in the form similar to the nonrelativistic case

$$
\phi_{\mathbf{P}}^{(r)}(\mathbf{K}, \mathbf{k})=\delta(\mathbf{P}-\mathbf{K}) \phi_{\mathrm{eff}}^{(r)}(\mathbf{p})
$$

and

$$
\begin{aligned}
\phi_{\mathbf{P}}^{(r)}(\mathbf{R}, \mathbf{r}) & =T_{\mathbf{R}} \phi_{\text {eff }}^{(r)}(\mathbf{r})=T_{\mathbf{R}} \frac{1}{(2 \pi)^{3 / 2}} \int\langle\mathbf{r} \mid \mathbf{k}\rangle \phi_{\text {eff }}^{(r)}(\mathbf{k}) \mathrm{d} \Omega \mathbf{k}= \\
& =\frac{1}{(2 \pi)^{3 / 2}} \int \mathrm{e}^{\mathrm{i} \mathbf{P R}}\langle\mathbf{r} \mid \mathbf{k}\rangle \phi_{\text {eff }}^{(r)}(\mathbf{k}) d \Omega \mathbf{k}
\end{aligned}
$$

or

$$
\phi_{\mathbf{P}}^{(r)}(\mathbf{R}, \mathbf{r})=\frac{1}{(2 \pi)^{3 / 2}} \int \mathrm{e}^{\mathrm{i} \mathbf{K} \mathbf{R}} \mathrm{e}^{\mathrm{ikr}} \phi_{\mathbf{P}}^{(r)}(\mathbf{K}, \mathbf{k}) \mathrm{d} \mathbf{K} \mathrm{d} \Omega \mathbf{k} .
$$

Now bilocal character of the 2-body wave function in contrast to the nonrelativistic case becomes essential because the variables $\mathbf{R}$, and $\mathbf{r}$ have the different nature.

\section{References}

[1] E. Wigner: Rev. of Mod. Phys. 21 (1949) 400.

[2] E. Wigner: Ann. of Math. 40 (1939) 149.

[3] A.S. Wightman: Rev. of Mod. Phys. 34 (1962) 845.

[4] M. I. Shirokov: Annalen der Physik 10 (1962) 60.

[5] R. M. Mir-Kasimov: Sov. Phys. JETP 22 (1966) 629.

[6] V. G. Kadyshevsky, R. M. Mir-Kasimov and N. B. Skachkov: Nuovo Cim. 55 (1968) 233.

[7] R. M. Mir-Kasimov: Journ. Phys. A. 24 (1991) 4283.

[8] R.M. Mir-Kasimov: Foundations of Physics 32 (2002) 607.

[9] Z. Can, Z. Güven, R. M. Mir-Kasimov and O. Oĝuz: Yadernaya Fizika (Physics of Atomic Nuclei) 64 (2001) 1985.

[10] H. C. Baehr, A. Dimakis and F. Müller-Hoissen: Journ. Phys. A 28 (1995) 3197.

[11] A. Dimakis and F. Müller-Hoissen: Jour. Math. Phys. (2003); 40 (1998) 1518.

[12] F. Bayen and J. Niederle: Czech. J. Phys. A 31 (1981) 1317.

[13] I. M. Gelfand and A.E. Shilov: Generalized functions and applications (Generalized Functions, vol.1), Academic Press, New-York, 1965.

[14] I. M. Gelfand, M. I. Graev and N. Ja. Vilenkin: Integral Geometry and Related Problems in the Theory of Representations (Generalized Functions, vol.5), Academic Press, New-York, 1966. 